Available online at:

https://acta-acustica.edpsciences.org

\title{
Influence of the multi-component electrical feed of air-core industrial reactors on their sound radiation
}

\author{
Paul Gning, Vincent Lanfranchi, and Nicolas Dauchez* \\ Sorbonne Universités, Université de Technologie de Compiègne, CNRS, FRE 2012 Roberval, Centre de recherche Royallieu, \\ CS 60 319, 60203 Compiègne cedex, France
}

Received 31 January 2020, Accepted 16 August 2020

\begin{abstract}
High voltage devices such as dry-type air-core reactors are subjected to environmental noise standards. Their excitation is due to Lorentz forces originated from the magnetic field, created by the coil itself, combined with the feed current. The objective of this paper is to show how spectral components present in the supply current of industrial dry-type air-core reactors is likely to produce a significant acoustic radiation. First, the multi-component distribution of the Lorentz forces is established. Then, the multi-physics computation process allowing to determine the acoustic pressure induced by each force component is presented. Finally, two industrial reactors are studied: a single and a multi-layer coil. It is pointed out that significant acoustic emergence can be induced by the interaction between small current spectral components with the fundamental or with each other.
\end{abstract}

\section{Introduction}

Dry-type air-core reactors used in electric grids can be source of noise pollution for neighborhood. The noise originates from the interaction of the electric current and the induced electromagnetic field, that produces periodic Lorentz forces exciting the structure [1]. These industrial reactors serve at limiting the fault currents, compensating the loads between two spectral components in parallel or filtering harmonics. Figure 1 shows an air-core reactor composed of four concentric coils fed in parallel through non-ferromagnetic spiders.

Electromagnetic generated noise has been the subject of research for several decades [2, 3]. Most of the literature focuses on electric machines, transformers or reactors with an iron core [4]. These devices include ferromagnetic materials that induce Maxwell's forces and magnetostriction phenomena. The vibrations and noise radiated are oftenly due to spectral components of electrical or magnetic origin. In the field of rotating machines, Le Besnerais et al. [5] developed an analytical multi-physics model relating multi-harmonics Maxwell forces to a vibroacoustic model. From experimental point of view, critical harmonics can be identified [6] to prevent mechanical damages. In the field of transformer, magnetostriction [7-9] and Maxwell forces [10] can be accounted in multi-physic models to predict the noise. A method to suppress some harmonics in windings of HVDC transformer is presented in [11].

\footnotetext{
*Corresponding author: nicolas.dauchez@utc.fr
}

Concerning air-core reactors, extensive work can be found on analytical methods to determine their electrical properties like the inductance $[12,13]$ or the AC resistance [14]. Some studies focus on the magnetic interactions between air-core coils [15-17]. However, to the knowledge of the authors, the vibroacoustic behavior of the air-core reactors is little studied. Lilien [18] proposed a simple electro-vibroacoustic analytic modeling to evaluate the generated noise of an air-core coil. The results were then compared with a numerical model. This approach was also used in a more recent study [19] with an improvement of the mechanical model and a calculation of Lorentz forces accounting for multi-components. The results obtained for a single layer air-core reactor are successfully compared with tests. However, these models are subject to hypotheses, like uniform magnetic field distribution, that do not allow accurate modeling of multi-layer reactors. Indeed, analytical methods are not well suited for a complex geometry such as an open finite size cylindrical shell [20, 21]. Recently, Gning [1] proposes a multi-physic model, coupling several numerical codes, to compute the sound radiation of a single-layer air-core coil fed by a purely sinusoidal current. Results agree well with measurements performed on a prototype coil intended for the manufacture of loudspeakers. It is shown that the breathing mode is the one producing the highest sound pressure level, and is dominating a large frequency range.

The objective of this paper is to show how the spectral components of the electric feed can combine with each other and interact with the structural modes to produce a 


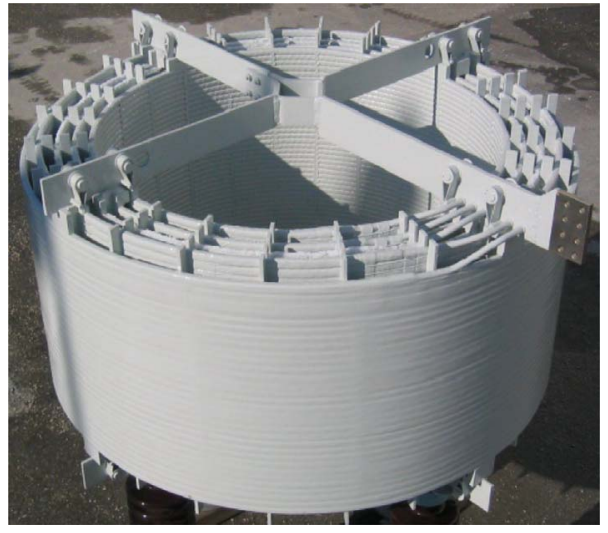

Figure 1. Industrial dry-type air-core reactor with four concentric layers.

significant acoustical response. Both single and multi-layers industrial dry-type air-core reactors are considered.

In the following, the effect of multi-components nature of the feed current on the spectrum of the Lorentz forces is illustrated through theoretical expressions and the computational method allowing to determine the acoustic response originating from the combination current components is described. Then, the sound radiation of two industrial air-core reactors, a single and a multi-layer, fed with such multi-components current is analyzed.

\section{Computational method}

\subsection{Derivation of multi-components Lorentz forces}

The supply of air-core reactors is an important factor in the study of their vibroacoustic behavior because the generated Lorentz forces depend on it. In an industrial context, the current is generally composed of a fundamental and numerous spectral components. Figure 2 shows two types of signals:

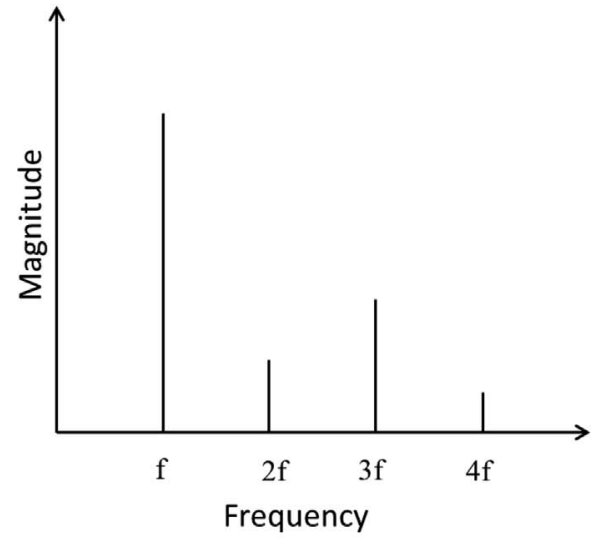

(a)
- an electrical network crossed by a current containing harmonics coming from nonlinear electric loads, each component being an integer multiple of the fundamental frequency $f$ as seen on Figure 2a,

- in the presence of Pulse Width Modulation (PWM), encountered when a reactor is connected to power electronics, there are spectral components around the switching frequency $f_{\mathrm{c}}$ and its multiples as illustrated in Figure $2 \mathrm{~b}$.

These harmonics or spectral components, coupled to the fundamental or between them, generate Lorentz forces at several frequencies which density at a given location writes:

$$
\begin{gathered}
d \vec{F}_{L}=\left\{\begin{array}{c}
0 \\
0 \\
J_{z h 0} \cos \left(\omega_{h 0} t+\varphi_{z h 0}\right)+J_{z h 1} \cos \left(\omega_{h 1} t+\varphi_{z h 1}\right)+J_{z h 2} \ldots
\end{array}\right\} \\
\times\left\{\begin{array}{c}
B_{x h 0} \cos \left(\omega_{h 0} t+\varphi_{x h 0}\right)+B_{x h 1} \cos \left(\omega_{h 1} t+\varphi_{x h 1}\right)+B_{x h 2} \cdots \\
B_{y h 0} \cos \left(\omega_{h 0} t+\varphi_{y h 0}\right)+B_{y h 1} \cos \left(\omega_{h 1} t+\varphi_{y h 1}\right)+B_{y h 2} \cdots \\
0
\end{array}\right\}\left\{\begin{array}{l}
\vec{x} \\
\vec{y} \\
\vec{z}
\end{array}\right\}
\end{gathered}
$$

where $(x, y, z)$ is the cartesian coordinate system, $h_{i}(i=1$, $2, \ldots)$ refers to the $i$ th spectral component for either the current or the flux density and $h_{0}$ refers to the fundamental component. By developing the previous formula, it comes:

$$
d \vec{F}_{L}=\left\{\begin{array}{c}
d F_{z h 0 \cdot y h 0}+d F_{z h 1 \cdot y h 0}+d F_{z h 0 \cdot y h 1}+d F_{z h 1 \cdot y h 1}+\ldots \\
d F_{z h 0 \cdot x h 0}+d F_{z h 1 \cdot x h 0}+d F_{z h 0 \cdot x h 1}+d F_{z h 1 \cdot x h 1}+\ldots \\
0
\end{array}\right\}\left\{\begin{array}{c}
\vec{x} \\
\vec{y} \\
\vec{z}
\end{array}\right\}
$$

where

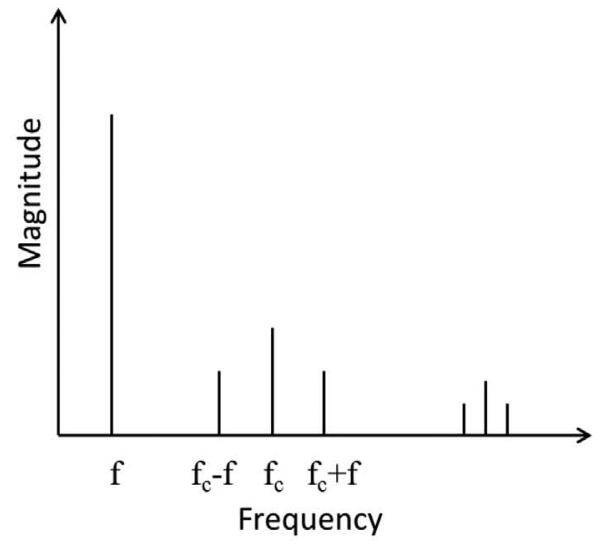

(b)

Figure 2. Typical current spectrum of an electrical grid with harmonic components (a) and with pulse width modulation (b). 


$$
\begin{gathered}
d F_{z h 0 \cdot y h 0}=-\frac{J_{z h 0} B_{y h 0}}{2}\left[\cos \left(\varphi_{y h 0}-\varphi_{z h 0}\right)\right. \\
\left.+\cos \left(2 \omega_{h 0} t+\varphi_{y h 0}+\varphi_{z h 0}\right)\right] \\
d F_{z h 1 \cdot y h 0}=-\frac{J_{z h 1} B_{y h 0}}{2}\left[\cos \left(\left(\omega_{h 0}-\omega_{h 1}\right) t+\varphi_{y h 0}-\varphi_{z h 1}\right)\right. \\
\left.+\cos \left(\left(\omega_{h 0}+\omega_{h 1}\right) t+\varphi_{y h 0}+\varphi_{z h 1}\right)\right] \\
d F_{z h 0 \cdot y h 1}=-\frac{J_{z h 0} B_{y h 1}}{2}\left[\cos \left(\left(\omega_{h 0}-\omega_{h 1}\right) t+\varphi_{z h 0}-\varphi_{y h 1}\right)\right. \\
\left.+\cos \left(\left(\omega_{h 0}+\omega_{h 1}\right) t+\varphi_{z h 0}+\varphi_{y h 1}\right)\right] \\
d F_{z h 1 \cdot y h 1}=-\frac{J_{z h 1} B_{y h 1}}{2}\left[\cos \left(\varphi_{z h 1}-\varphi_{y h 1}\right)\right. \\
\left.+\cos \left(2 \omega_{h 1} t+\varphi_{z h 1}+\varphi_{x h 1}\right)\right] .
\end{gathered}
$$

The forces $d F_{z h 0 \cdot x h 0}, d F_{z h 1 \cdot x h 0}, d F_{z h 0: x h 1}$ and $d F_{z h 1 \times x h 1}$ can be obtained the same way by substituting $y$ by $x$. Multiple spectral components of forces are created at different frequencies. For a signal composed of two components of pulsation $\omega_{\mathrm{h} 0}$ and $\omega_{\mathrm{h} 1}$, the spectrum of the Lorentz forces contains components at the following frequencies: $2 \omega_{h 0}$, $2 \omega_{h 1}, \omega_{h 0}-\omega_{h 1}$ and $\omega_{h 0}+\omega_{h 1}$. Note that in addition to the double frequencies components, two spectral components specifically arise from the interaction between the current and induction spectral components at different frequencies.

\subsection{Numerical computation process for multi- components force spectrum}

The multi-physic computational method presented in our previous work [1] is based on a numerical process considering a single harmonic component. The process consists in coupling electromagnetic, mechanical and vibroacoustic numerical models within four steps as shown in Figure 3:

- Step 1 is an electromagnetic calculation using Altair Flux3D software, that solves the Maxwell equations. The input is the electric current density $\mathbf{J}$ and the output is Lorentz force density $d \mathbf{F}_{L}$ in the volume of the coil's conductor. The results are computed in harmonic regime which means at a single frequency component.

- Step 2 is the calculation of the vibration modal basis $\boldsymbol{\Theta}_{m}(x, y, z)$ with MSC Nastran software.

- Step 3 is the projection of the Lorentz forces on the modal basis to get the modal forces:

$$
F_{\mathrm{Lm}}=\int_{V} \boldsymbol{\Theta}_{m}(x, y, z) \cdot d \mathbf{F}_{L}(x, y, z) d V
$$

- Step 4 performs a coupled structural and acoustic calculation by boundary element method in ESI VaOne $[10,22]$ using of the modal basis and forces computed in Steps 2 and 3.

To manage a multi-component electrical spectrum, two options are available. First, the calculation of Lorentz forces can be managed considering the multi-component electrical current in Flux3D operating in transient mode. The output is a multi-component force spectrum. Steps 3 and 4 are then repeated for each force component. The second option is to treat each component separately in Flux3D: a preliminary step is necessary (see Step 0 in Fig. 3) for the components created by the interaction between a current and an induction spectral components, at frequency $\omega_{h 0}-\omega_{h 1}$ and $\omega_{h 0}+\omega_{h 1}$ for instance, to determine the equivalent input current to get the same force density magnitude as the one obtained with the real multi-harmonic current. According to equation (2), the interaction of the fundamental and a given component leads to a force density which magnitude is given by the sum of two contributions:

$$
d F=\frac{J_{z h 0} B_{(x, y) h 1}}{2}+\frac{J_{z h 1} B_{(x, y) h 0}}{2} .
$$

This expression results from the interaction of fundamental current $h 0$ and harmonic $h 1$ of the induction and conversely that of current harmonic $h 1$ and the fundamental induction $h 0$. Knowing that induction is proportional to current density, the previous expression can be written:

$$
d F=k J_{z h 0} J_{z h 1},
$$

where $k$ is the coefficient between current density and induction.

If a single frequency component is considered, the force density has a single term $d F=\frac{J_{z} B_{(x, y)}}{2}$. Using the same coefficient $k$ between the current density and the induction, we have

$$
d F=\frac{k J_{\text {eq }}^{2}}{2},
$$

where $J_{\text {eq }}$ stands for the equivalent current. To get the equality between equations (4) and (5), we must have

$$
J_{\text {eq }}=\sqrt{2 J_{z h 0} J_{z h 1}} \text {. }
$$

Finally, since the current density $J$ is proportional to the current magnitude $I$, to predict the force generated by a fundamental current supply combined with a component of magnitudes $I_{h 0}$ and $I_{h 1}$ respectively, the equivalent current magnitude $I_{\mathrm{eq}}$ is determined using the same equation

$$
I_{\text {eq }}=\sqrt{2 I_{h 0} I_{h 1}} \text {. }
$$

As an example, to predict the force generated by a fundamental current supply of 450 A RMS combined with a component of $2.25 \mathrm{~A}$ RMS $(0.5 \%$ of fundamental), the equivalent current $I_{\mathrm{eq}}=\sqrt{2 \times 450 \times \frac{0.5 \times 450}{100}}=45 \mathrm{~A}$ is used in the single frequency analysis tool at half the frequency of the generated Lorentz force that is $\left(\omega_{h 0}-\omega_{h 1}\right) / 2$ and $\left(\omega_{h 0}+\omega_{h 1}\right) / 2$.

\section{Sound radiation of a single layer air-core reactor}

This section presents the computation process applied to the single layer air-core coil named Coil55 and shown in Figure 4. Its characteristics are given in Table 1. First, 


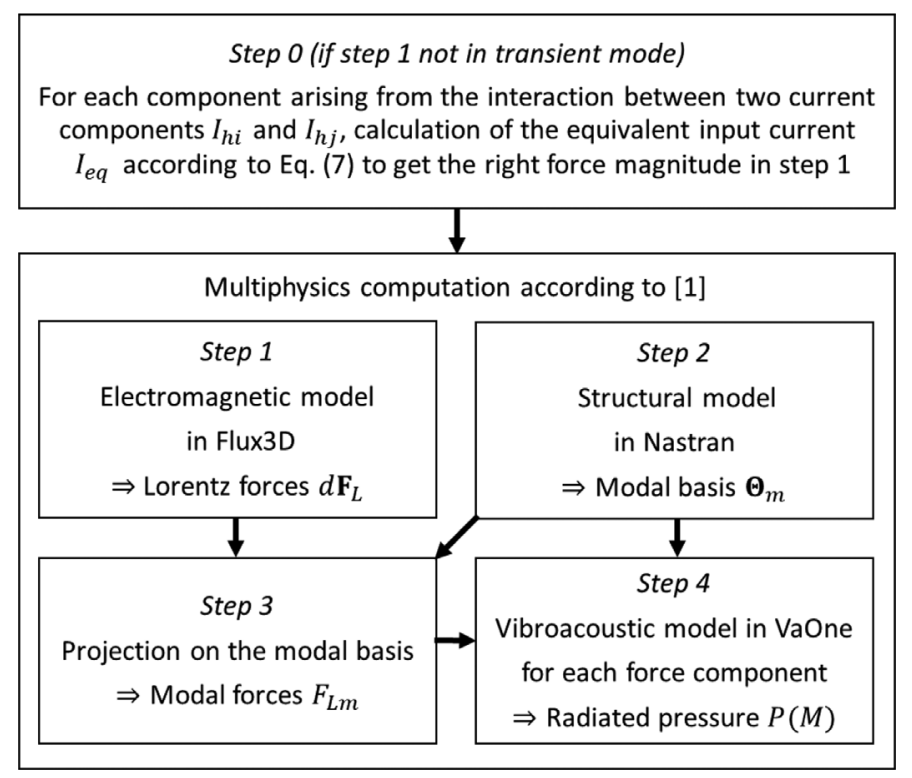

Figure 3. Computation process for a multi-components input current.

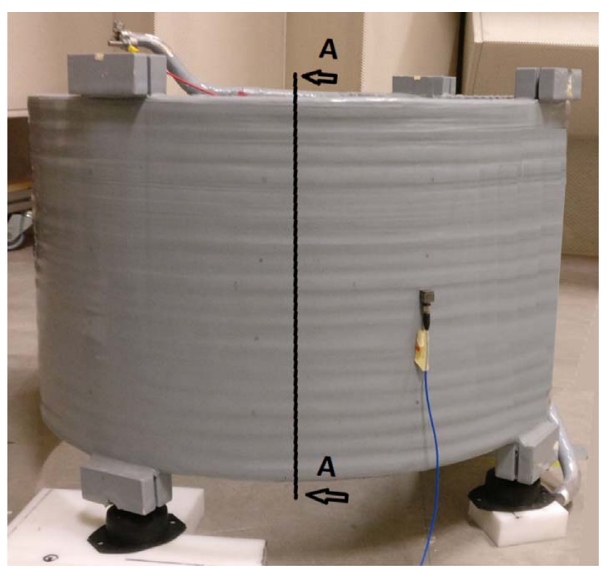

Figure 4. Experimental set-up for Coil55 modal analysis.

Table 1. Characteristics of Coil55 reactor.

\begin{tabular}{lcc}
\hline Nominal current & 450 & $\mathrm{~A}$ \\
Inductance & 0.17 & $\mathrm{mH}$ \\
Inner diameter & 553 & $\mathrm{~mm}$ \\
Height & 360.5 & $\mathrm{~mm}$ \\
Radial thickness & 24.2 & $\mathrm{~mm}$ \\
\hline
\end{tabular}

electromagnetic and structural properties are investigated and compared with experimental results. Then the acoustic response of the coil powered with a current carrying a given spectral component is computed. The objective is to show how a small magnitude component can induce a significant acoustical response.

\subsection{Electromagnetic properties}

The flux density given by the electromagnetic model of Coil55 using Altair Flux3D software is compared with experiments. The coil is fed with a current of 6 A RMS. The RMS values of the flux density are shown in Figure $5 \mathrm{a}$. Figure 6 shows that the values taken at five points inside the coil (see Fig. 5a) are in good agreement with measurements obtained with a teslameter. Figure $5 \mathrm{~b}$ shows the Lorentz forces induced by the interaction between the flux density and the current: since the flux density is not homogeneous in the coil, Lorentz forces vary in magnitude and direction within the coil section.

\subsection{Mechanical properties}

The computation of the modal basis of the structure is the step 2 of the simulation process (see Fig. 3). The mechanical properties used for Coil55 are derived experimentally. First, an experimental modal analysis is performed: the coil is excited with a shock hammer B\&K 8206 over 12 circumferential positions set at the median height of the coil, and the response is collected with an accelerometer B\&K 4507 at a single position (see Fig. 4). The coil is placed on three soft pads that have been designed so that the boundary conditions can be assumed free. The measurement acquisition and the modal analysis are performed within B\&K Pulse Labshop environment. The experimental modal shape and their frequencies are given in Figure 7. Radial modes with 2, 3 or 4 lobes are clearly observed at $126 \mathrm{~Hz}, 355 \mathrm{~Hz}$ and $667 \mathrm{~Hz}$ respectively. The breathing mode is observed at $2455 \mathrm{~Hz}$.

The numerical modal basis is computed by finite element using MSC Nastran software. The elastic properties of the coil, given in Table 3, have been adjusted to better fit the experimental results. The isotropic properties, close to standard aluminum, allow to get the frequency of mode $(0,0)$ in adequacy with measurement as shown in Table 2. However for the radial bending modes the discrepancy is around $24 \%$. Anisotropic properties, adjusted to get better 


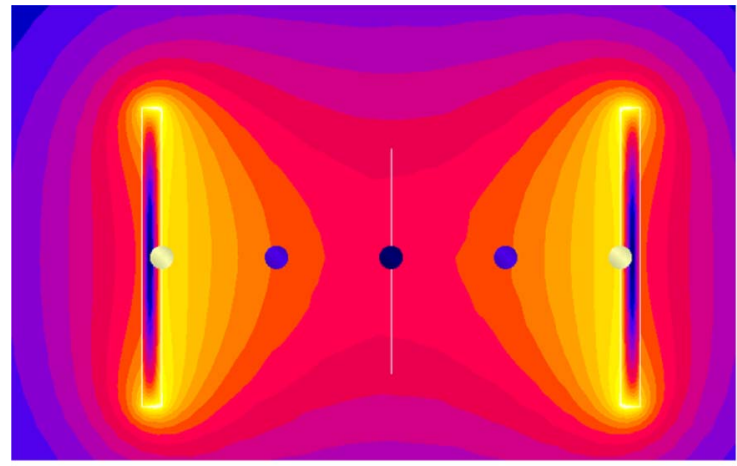

(a)

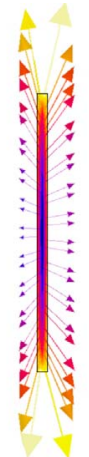

(b)

Figure 5. (a) Flux density and position of the 5 points where measurements are compared with the simulation and (b) Lorentz forces $d F_{L}$ distribution within the section A-A of the Coil55 (see Fig. 4).

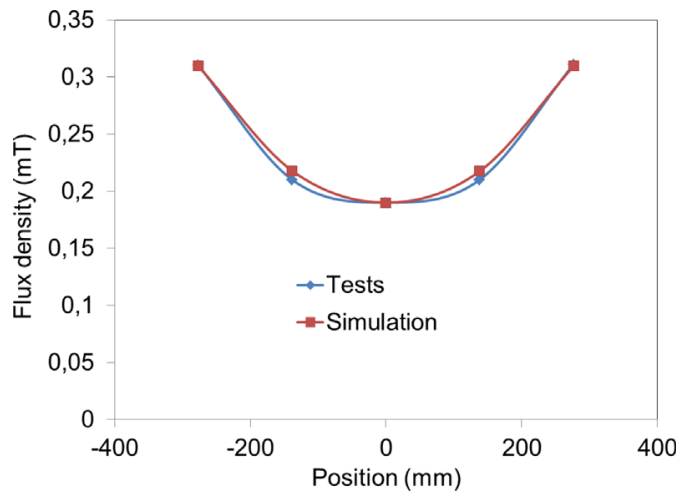

Figure 6. Comparison of the simulated and measured flux density inside the reactor Coil55. (a)

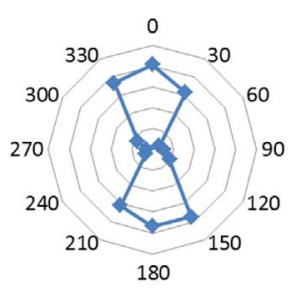

(c)

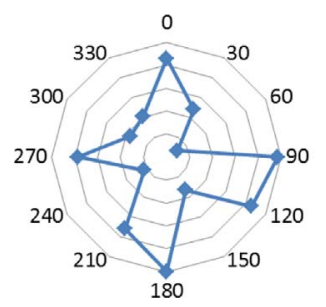

(b)

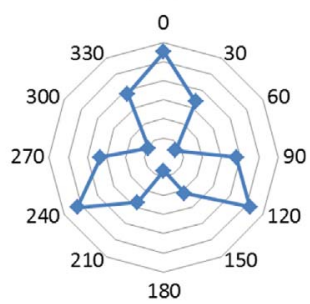

(d)

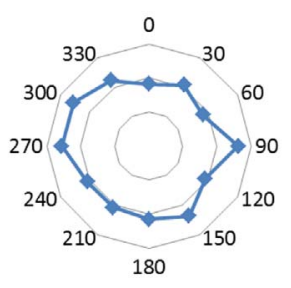

Figure 7. Experimental mode shapes of Coil55: $(2,0)$ at $126 \mathrm{~Hz}$ (a), $(3,0)$ at $355 \mathrm{~Hz}(\mathrm{~b})$, at $667 \mathrm{~Hz}(4,0)(\mathrm{c})$ and $(0,0)$ at $2455 \mathrm{~Hz}$ (d).

results, will then be used in the following. These properties are those of a material equivalent to the multi-strand aluminium cable as shown in Figure 8, where the tangential direction $T$, oriented along the strands, is assumed to be an axis of symmetry.

\subsection{Simulation of the acoustic response under multi- component current supply}

In this section, we consider the acoustic response of the Coil55 fed with its nominal current of RMS value $I=450 \mathrm{~A}$ and a frequency of $50 \mathrm{~Hz}$, carrying a spectral component of $0.5 \%$ of the fundamental magnitude at the frequency of $2385 \mathrm{~Hz}$. This frequency is chosen to produce Lorentz force components close to the frequency of the breathing mode $(0,0)$. According to equation (2), the Lorentz forces can be written in the following form

$$
\begin{aligned}
F_{L}= & F_{0}+F_{1} \cos (2 \pi \times 100 t)+F_{2} \cos (2 \pi \times 2335 t) \\
& +F_{3} \cos (2 \pi \times 2435 t)+F_{4} \cos (2 \pi \times 4770 t),
\end{aligned}
$$

where $F_{\mathrm{i}}$ are the magnitude of each force component given in Table 4. They are obtained with Flux3D used in transient mode, allowing to account for multiple frequencies. The maximum magnitude is for the DC component at $0 \mathrm{~Hz}$ coming from the frequency combination (50-50) and (2385-2385). The other components derive from the following combinations: $100 \mathrm{~Hz}(50+50), 2335 \mathrm{~Hz}$ $(2385-50), 2435 \mathrm{~Hz}(2385+50)$ and $4770 \mathrm{~Hz}$ $(2385+2385)$. As the force component $F_{3}$ at $2435 \mathrm{~Hz}$ coincides with the frequency of the mode $(0,0)$, a resonance is likely to occur. To quantify the effect of this component, the equivalent current is computed according to equation (7), giving $I_{\text {eq }}=45 \mathrm{~A}$ RMS. The computed Lorentz force is projected on the modal basis. Figure 9a shows that a very good spatial mode matching with breathing mode $(0,0)$ is observed. Due to the match in shape and frequency between $F_{3}$ force component and the breathing mode, a significant noise level around $62 \mathrm{~dB}$ at $1 \mathrm{~m}$ from the reactor is produced. Corresponding acoustic field obtained is presented in Figure 10.

To get a more general idea on the acoustic effect of a small current component, a sweep in frequency with $F_{3}$ force magnitude is carried out. Figure $9 \mathrm{~b}$ shows the acoustic response of the reactor at $1 \mathrm{~m}$ distance. Mode $(0,0)$ is clearly 
Table 2. Frequencies in $\mathrm{Hz}$ of Coil55 modes obtained by test (Exp.) and simulations (Sim.) with isotropic and anisotropic properties, and the relative difference (Diff.) with experiment in \%.

\begin{tabular}{lccccc}
\hline Modes & Exp. & Sim. isotropic & Diff. isotropic & Sim. anisotropic & Diff. anisotropic \\
\hline$(0,0)$ & 2455 & 2435 & $-1 \%$ & 2467 & 147 \\
$(2,0)$ & 126 & 166 & $24 \%$ & 377 & $14 \%$ \\
$(3,0)$ & 355 & 467 & $24 \%$ & 647 & $6 \%$ \\
$(4,0)$ & 667 & 888 & $25 \%$ & 933 & $-3 \%$ \\
$(5,0)$ & 1086 & 1423 & $24 \%$ & $-16 \%$ \\
\hline
\end{tabular}

Table 3. Material properties used for the computation of the modal basis of Coil55: $E$ is the Young modulus in GPa, $G$ is the shear modulus in GPa and $v$ is the Poisson's ratio. Orientations are given by $T$ (tangential $=$ direction along the strands), $L$ (longitudinal $=$ coil axis) and $R$ (radial).

\begin{tabular}{lcc}
\hline & Isotropic & Anisotropic \\
\hline $\mathrm{E}_{\mathrm{T}}$ & 70 & 70 \\
$\mathrm{E}_{\mathrm{L}}$ & 70 & 40 \\
$\mathrm{E}_{\mathrm{R}}$ & 70 & 40 \\
$\mathrm{G}_{\mathrm{TL}}$ & 25 & 0.78 \\
$\mathrm{G}_{\mathrm{LR}}$ & 25 & 25 \\
$\mathrm{G}_{\mathrm{RT}}$ & 25 & 25 \\
$v_{\mathrm{TL}}$ & 0.3 & 0.1 \\
$v_{\mathrm{LR}}$ & 0.3 & 0.1 \\
$v_{\mathrm{RT}}$ & 0.3 & 0.1 \\
\hline
\end{tabular}

preponderant in the acoustic response. Below its frequency, the responses of radial bending modes are shown to be also quite significant. Indeed, a relevant projection of the force shape on radial bending modes $(2,0),(3,0),(4,0)$ and $(7,0)$ can also be observed in Figure 9a. Figure 9b also shows that, as opposed as mode $(0,0)$ which is axis-symmetrical, radial bending modes induce an angle dependent acoustic response. By the way, the acoustic response mode $(0,0)$ only depends on the distance from the reactor in the $(X, Z)$ plane as show in Figure 10.

To sum up, a current spectral component as little as $0.5 \%$ of the nominal current can create, by interaction with the fundamental current, a force exciting structural modes, producing a significant sound radiation.

\section{Sound radiation of a multi-layer industrial reactor}

In this section, a multi-layer industrial reactor used on a power grid, of the type presented in Figure 1, is considered. It is composed of eight concentric coils separated by an air gap of about $45 \mathrm{~mm}$. The height is $2989 \mathrm{~mm}$, the inner diameter $1318 \mathrm{~mm}$ and the outer diameter $1976 \mathrm{~mm}$. This reactor, being not available for testing in our laboratory, only a numerical analysis is presented.

\subsection{Mechanical properties and input current spectrum}

The structural behavior of the multi-layer reactor is characterized by its modal basis computed at step 2 with MSC Nastran software. The isotropic properties of the

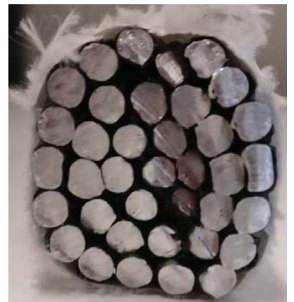

Figure 8. View of Coil55 cable section composed of 37 aluminum strands.

Table 4. Magnitude of the main Lorentz force components.

\begin{tabular}{lcccc}
\hline & $F_{0}$ & $F_{1}$ & $F_{2}$ & $F_{3}$ \\
\hline Frequency $(\mathrm{Hz})$ & 0 & 100 & 2335 & 2435 \\
Magnitude $\left(\mathrm{N} \mathrm{m}^{-3}\right)$ & 248 & 115 & 3.3 & 3.3 \\
\hline
\end{tabular}

material given in Table 3 are considered. The spiders and spacers as shown in Figure 1 are not accounted for in the model since it has been shown that they have a little influence. Figure 11 shows four of the most radiating mode shapes. The frequency of the first radial mode $(2,0)$ and breathing mode $(0,0)$ are respectively $10 \mathrm{~Hz}$ and $737 \mathrm{~Hz}$.

In service, this reactor is submitted to a $1000 \mathrm{~A}$ RMS quasi-sinusoidal current composed of a fundamental at $50 \mathrm{~Hz}$ and several harmonics. Since the load of the reactor is permanently varying with service conditions, the current harmonics magnitudes are time dependent. An onsite measurement for a given load gives the relative magnitudes of the first 13 harmonics summarized in Table 5: most of the magnitudes are between $1 \%$ and $2 \%$ of the fundamental. The combination of these harmonics with the fundamental frequency and between them is likely to create a wide range of frequency components, up to $2 \times 13^{2}$ Lorentz force components in the frequency range from $0 \mathrm{~Hz}$ to $1300 \mathrm{~Hz}$.

\subsection{Sound radiation as a function of frequency}

The sound radiation of the industrial reactor is first studied as a function of the frequency. The acoustic response is computed considering a single force magnitude on the whole frequency range. An equivalent current of 100 A RMS magnitude is considered. This corresponds to an interaction between fundamental current at $1000 \mathrm{~A}$ RMS and a spectral component of $0.5 \%$ relative magnitude according to equation (7). It should be noted that a constant structural damping of $2 \%$ is considered. The acoustic 


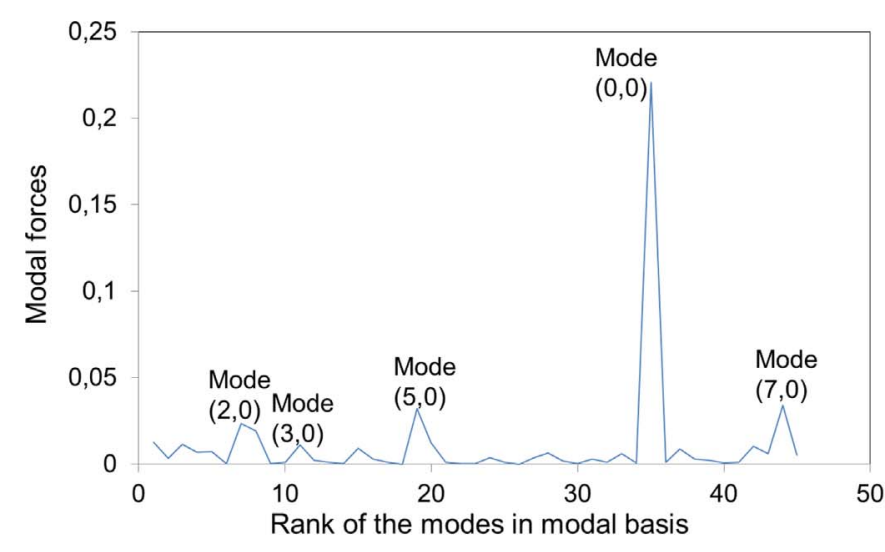

(a)

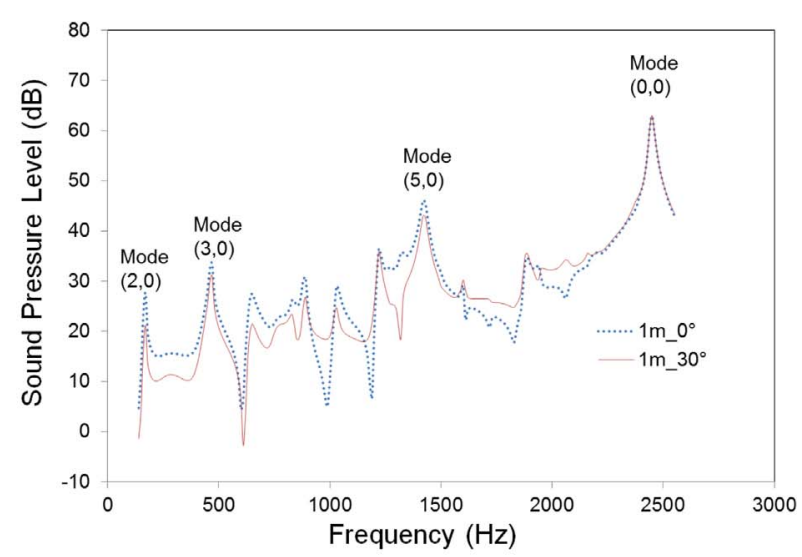

(b)

Figure 9. Modal forces in N (a) and simulated acoustic response of Coil55 at $1 \mathrm{~m}$ for a 45 A RMS single component (b).
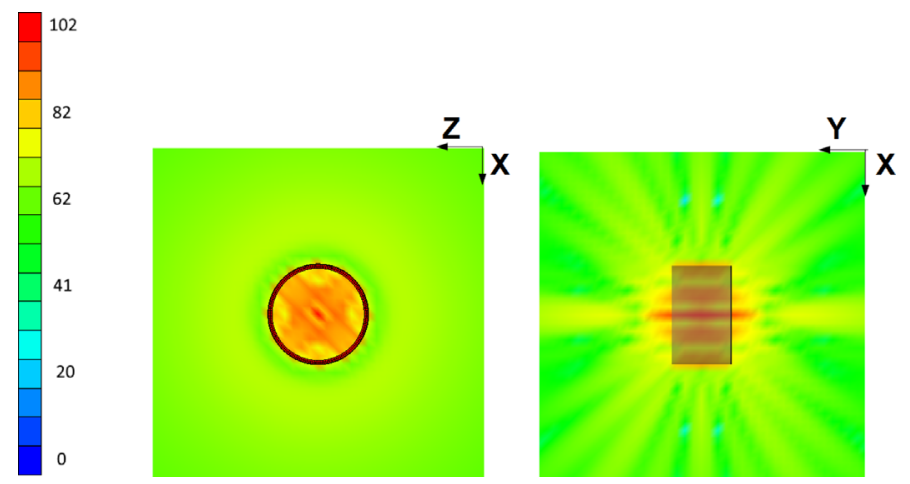

Figure 10. Acoustic response map in $\mathrm{dB}$ at the frequency of mode $(0,0)$ of Coil55.

response is given in Figure 12. The overall noise level is 60 $\mathrm{dB}$ at $10 \mathrm{~m}$ from the coil with a maximum level of $57 \mathrm{~dB}$ at the frequency of mode $(0,0)$. The contribution of the other modes such as radial bending modes are noticeable and tends to be generally more significant when they get closer to the frequency of mode $(0,0)$.

\subsection{Sound radiation for multi-components current supply}

In this section, we focus on the spectral components that are close to structural modes, so that they can induce a structural resonance producing a significant acoustic level.

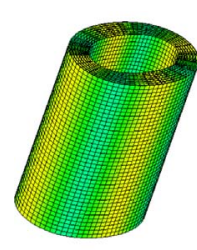

(a)

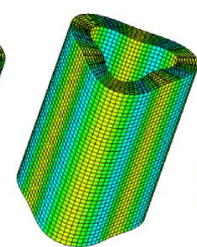

(b)

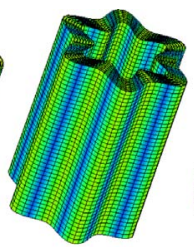

(c)

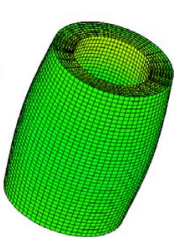

(d)
Figure 11. Mode shapes of the multi-layer reactor. (a) mode $(2,0)$ at $10 \mathrm{~Hz}$; (b) mode $(3,0)$ at $29 \mathrm{~Hz}$; (c) mode $(6,0)$ at $134 \mathrm{~Hz}$; (d) mode $(0,0)$ at $737 \mathrm{~Hz}$.
The onsite measured current harmonics presented at Table 5 are considered to derive the Lorentz force components.

The radial bending mode $(6,0)$ at $134 \mathrm{~Hz}$ could be efficiently excited by the first Lorentz force component occurring at $100 \mathrm{~Hz}$. This force component is generated by the combination of fundamental current and flux density which is the maximum magnitude component. The acoustic level

Table 5. Current harmonics as a percentage of the fundamental (measured on site).

\begin{tabular}{lc}
\hline Rank & Current magnitude $(\%)$ \\
\hline $1(50 \mathrm{~Hz})$ & 100 \\
2 & 1.5 \\
3 & 2 \\
4 & 0.8 \\
5 & 3.5 \\
6 & 0.2 \\
7 & 1.8 \\
8 & 0 \\
9 & 0.3 \\
10 & 0 \\
11 & 1.2 \\
12 & 1.4 \\
13 & 1 \\
\hline
\end{tabular}




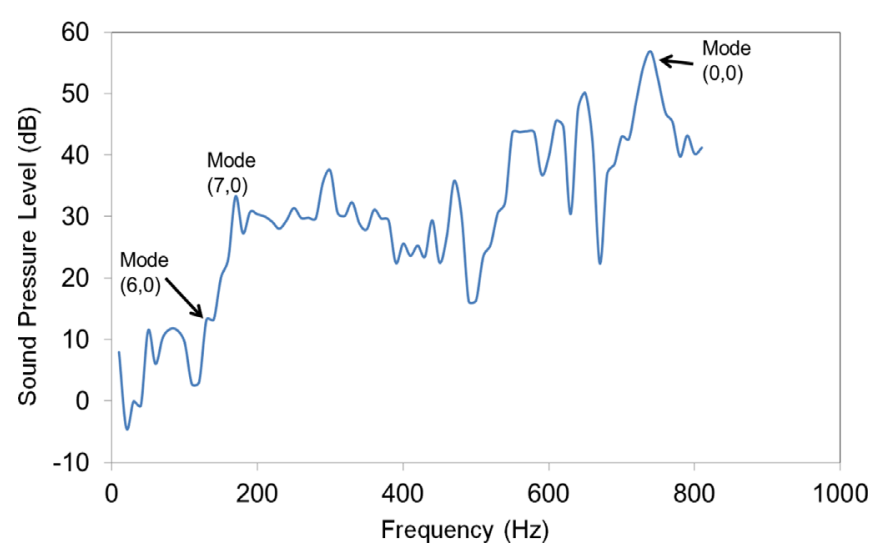

Figure 12. Acoustic response of the multi-layer reactor at a distance of $10 \mathrm{~m}$.

at $10 \mathrm{~m}$ is $35 \mathrm{~dB}$ at $100 \mathrm{~Hz}$. Note that this is the maximum level as a function of the angle.

The mode $(7,0)$, which natural frequency is around $185 \mathrm{~Hz}$, could be excited by the interaction between the fundamental and harmonic 2 at a frequency of $150 \mathrm{~Hz}$. Its magnitude is $1.5 \%$ of the fundamental. In this case, the noise level at $10 \mathrm{~m}$ and $150 \mathrm{~Hz}$ is $40 \mathrm{~dB}$.

Finally, the breathing mode $(0,0)$ at $737 \mathrm{~Hz}$ could be excited by the spectral component resulting from the combination between the fundamental at $50 \mathrm{~Hz}$ and the harmonic 13 at $650 \mathrm{~Hz}$ for a response at $700 \mathrm{~Hz}$. The magnitude of harmonic 13 is $1 \%$ of the fundamental. The level obtained at $10 \mathrm{~m}$ is $54 \mathrm{~dB}$. If both frequencies coincide, the level would rise up to $69 \mathrm{~dB}$ due to resonance effect. The damping of this mode is, by experience, lower than those of the radial bending modes. So assuming that the damping is $1 \%$ instead of $2 \%$, predicted noise level may increase up to $75 \mathrm{~dB}$ at $10 \mathrm{~m}$. The breathing mode $(0,0)$ is definitely the most contributing to the sound level radiated by the coil, originated by the interaction between the fundamental and a high order harmonic component.

\section{Conclusion}

In this paper, the multi-components electric feed of industrial dry-type air-core reactors has been considered on their acoustical radiation. The interaction between the current components and the induced magnetic field components lead to a higher number of Lorentz force component: a current spectrum having the fundamental and one harmonic will generate a force spectrum having four components.

To derive the acoustical radiation field, a multi-physic computation considering each component apart is necessary. For each components, an equivalent input current is first calculated.

Two industrial reactors has been studied: a single and a multi-layer having eight concentric layers. Their mechanical properties have been ajusted to fit their modal behavior. For both configuration, it is shown that the combination of the fundamental with one harmonic components having a relative level less than $1 \%$ can induce a significant acoustical response of the breathing mode. Moreover for both reactors, the frequency of the breathing mode is close to the maximum sensitivity of the human ear that is around $1 \mathrm{kHz}$. Radial bending modes at lower frequencies can also induce a significant acoustical response but in a lesser extend, since the Lorentz force spatial distribution is less effective to excite non axisymmetrical modes.

In conclusion, the high number of harmonics present in the electrical feed is willing to produce a significant sound level by exciting numerous structural modes, the breathing mode being the more effective. Moreover, considering harmonics magnitude variability in function of time, it is possible that the noise radiated by the reactor becomes intermittently very high.

Future work deals with the optimization of industrial reactors design and their implementation to meet environmental standards.

\section{Acknowledgments}

The authors would like to thank RTE for funding and participating to this research work.

\section{Conflict of interest}

The authors declare that they have no conflict of interest.

\section{References}

1. P. Gning, V. Lanfranchi, N. Dauchez, K. Bouayed: Multiphysics model for predicting the sound radiation of a singlelayer air-core coil. Applied Acoustics 146 (2019) 327-333.

2. J.F. Gieras, Z.J. Wang, J.C. Lai: Noise of polyphase electric motors, CRC Press, 2005.

3. A. McCloskey, X. Arrasate, X. Hernandez, I. Gomez, G. Almandoz: Analytical calculation of vibrations of electromagnetic origin in electrical machines. Mechanical Systems and Signal Processing 98 (2018) 557-569.

4.F. Devaux, S. Ryder, M. Rosner: Bruit généré par les transformateurs et bobines d'inductances : différents types et différentes solutions (Noise generated by transformers and coils: different kinds and solutions). Alstom T\&D, 2003.

5. J.Le Besnerais, A. Fasquelle, M. Hecquet, J. Pelle, V. Lanfranchi, S. Harmand, P. Brochet, A. Randria: Multiphysics modeling: Electro-vibro-acoustics and heat transfer of PWM-Fed induction machines. IEEE Transactions on Industrial Electronics 57 (2010) 1279-1287.

6. S. Baldizzone, C.J. Novak, N.C. Kar: Experimental investigations of noise and vibration in electric machines, in: IEEE Transportation Electrification Conference and Expo (ITEC), 2012, pp. 1-6.

7.S.S. Mbengue, N. Buiron, V. Lanfranchi: Macroscopic modeling of anisotropic magnetostriction and magnetization in soft ferromagnetic materials. Journal of Magnetism and Magnetic Materials 404 (2016) 74-78.

8. P. Shuai, J. Biela: Impact of core shape and material on the acoustic noise emission of medium frequency, medium voltage transformers, in: 7th European Conference on Power 
Electronics and Applications (EPE'15 ECCE-Europe), 2015, pp. $1-11$.

9. S.S. Mbengue, N. Buiron, V. Lanfranchi: An anisotropic model for magnetostriction and magnetization computing for noise generation in electric devices. Sensors 16 (2016) 553.

10. K. Bouayed, L. Mebarek, V. Lanfranchi, J.-D. Chazot, R. Marechal, M.-A. Hamdi: Noise and vibration of a power transformer under an electrical excitation. Applied Acoustics 128 (2017) 64-70.

11. P. Shao, L. Luo, Y. Li, C. Rehtanz: Electromagnetic vibration analysis of the winding of a new HVDC converter transformer. IEEE Transactions on Power Delivery 27, 1 (2012) 123-130.

12. J.T. Conway: Inductance calculations for circular coils of rectangular cross section and parallel axes using Bessel and Struve Functions. IEEE Transactions on Magnetics 46, 1 (2010) 75-81.

13. Y. Luo, B. Chen: Improvement of self-inductance calculations for circular coils of rectangular cross section. IEEE Transactions on Magnetics 49, 3 (2013) 1249-1255.

14. F. Chen, X. Ma, Y. Zhao, J. Zou: Support vector machine approach for calculating the $\mathrm{AC}$ resistance of air-core reactor. IEEE Transactions on Power Delivery 26, 4 (2011) $2407-2415$.

15. S. Babic, F. Sirois, C. Akyel, G. Lemarquand, V. Lemarquand, R. Ravaud: New formulas for mutual inductance and axial magnetic force between a thin wall solenoid and a thick circular coil of rectangular cross-section, IEEE Transactions on Magnetics 47, 8 (2011) 2034-2044.

16.Z.J. Wang, Y. Ren: Magnetic force and torque calculation between circular coils with nonparallel axes. IEEE Transactions on Applied Superconductivity 24, 4 (2014) 1-5.

17. R. Ravaud, G. Lemarquand, S. Babic, V. Lemarquand, C. Akyel: Cylindrical magnets and coils: fields, forces, and inductances. IEEE Transactions on Magnetics 46, 9 (2010) 3585-3590.

18. J.-L. Lilien: Acoustic noise generated by air power reactor in open-air substation. European Transactions on Electrical Power 16, 3 (2006) 297-310.

19. T.A. Fiorentin, L.F. Lopes, O. Mecias da Silva Jr., A. Lenzi: Vibroacoustic models of air-core reactors. The International Journal of Acoustics and Vibration 21, 4 (2016) 453-461.

20. O. Unruh: Parametric study of sound radiation properties of complex vibration patterns in rectangular plates using an analytical model. Acta Acustica 101 (2015) 701-712.

21. D. Fritze, S. Marburg, H.-J. Hardtke: Estimation of radiation sound power: A case study on common approximation methods. Acta Acustica 95 (2009) 833-842.

22. J.B. Mariem, M.A. Hamdi: A new boundary finite element method for fluid-structure interaction problems. International Journal for Numerical Methods in Engineering 24, 7 (1987) 1251-1267.

Cite this article as: Gning P, Lanfranchi V \& Dauchez N. 2020. Influence of the multi-component electrical feed of air-core industrial reactors on their sound radiation. Acta Acustica, 4, 14. 